\title{
ASSOCIAÇÃO SINGULAR DE SÍNDROME DE KALLMANN E CISTO ARACNÓIDE DA FOSSA MÉDIA
}

\author{
RELATO DE CASO
}

\author{
VVENS B. FERNANDES *, GIL GUERRA JR**, SOFIA H. V. LEMOS**, MARIA T. MATIAS ${ }^{* *}$, \\ BENITO P. DAMASCENO*, OSMI HAMAMOTO*, ARY MARCONI JR*, \\ DONIZETTI C. HONORATO *, JOSÉ L. C. MARINS ***
}

\begin{abstract}
RESUMO - O hipogonadismo hipogonadotrófico pode resultar de diferentes anomalias do sistema nervoso central, apresentando sinais clínicos que dependem da idade de aparecimento, bem como do grau de deficiência gonadotrófica e de sua associação com outras deficiências hipofisárias. Relatamos o caso de um rapaz de 18 anos com atraso puberal, retardo de crescimento estatural a partir de 10 anos e história de dificuldade de aprendizado escolar. Ao exame apresentava discreto aspecto eunucóide, $162 \mathrm{~cm}$ de altura $(\mathrm{z}$ score $=-2,17)$, estadiamento puberal GII, PII, testículos de $4 \mathrm{~cm}^{3}$. A avaliação complementar demonstrou níveis pré-puberais de testosterona, megateste com resposta normal, exceto ao teste de estímulo com GnRH agudo e prolongado. CT de crânio mostrou cisto aracnóide da fossa média esquerda com extensāo supra-selar. Foi feito o diagnóstico de hipogonadismo hipogonadotrófico provavelmente secundário à compressão pelo cisto aracnóide e por isso, optou-se por derivação cisto-peritoneal. Após a cirurgia não houve retomada do desenvolvimento puberal, sendo entāo verificada anosmia bilateral e, portanto, diagnosticada Síndrome de Kallmann, que foi confirmada pelos achados da RNM, embora os resultados hormonais não sejam totalmente compatíveis com a referida síndrome. Não encontramos na literatura descrição da associação entre Síndrome de Kallmnann e cisto aracnoide e acreditamos que neste caso os resultados da avaliação hormonal possam ser devidos a tal associação que provocou adicionalmente uma disfunção hipofisária.
\end{abstract}

PALAVRAS-CHAVE: hipogonadismo, cisto aracnóide, Síndrome de Kallmann.

\section{Singular association of Kallmann's syndrome and arachnoid cyst of middle fossa: case report}

SUMMARY - Hypogonadotrophic hypogonadism can result from different abnormalities in the central nervous system. The clinical picture depends upon the time of onset the deficiency, the magnitude of the gonadotropins deficiency and whether there are other pituitary hormone deficiencies as well. We report on a 18-year-old boy, who was investigated because of pubertal and growth delay. He also had learning disabilities. On physical examination he exhibited mild eunuchoid aspect, $162 \mathrm{~cm}$ height $(\mathrm{z}$ score $=-2,17$ ), pubertal development on stage G II, P II, and $4 \mathrm{~cm}^{3}$ testis. Laboratory investigation revealed pre-pubertal levels of testosterone and normal results of the combined test of anterior pituitary function, except for in GnRH acute and prolonged test. Brain CT showed an arachnoid cyst on left middle fossa with expansion to suprasellar cisterna. He was diagnosed as having hypogonadotrophic hypogonadism secondary to compression by the cyst, and a cyst-peritoneal derivation was performed. After surgery there was no improvement of the pubertal state and bilateral anosmia was discovered, so Kallmann's syndrome was then diagnosed and was confirmed by MRI, even though the hormonal results are not totally matched with the refered syndrome. We did not find in the literature any description of the association between Kallmann's syndrome and arachnoid cyst and we believe that in this case the results of the hormonal measurement may be due to such association that provoked an additional hypophysis dysfunction.

KEY WORDS: hypogonadism, arachnoid cyst, Kallmann's Syndrome.

Estudo realizado na Faculdade de Ciências Médicas (FCM) da Universidade Estadual de Campinas (UNICAMP): * Disciplina de Neurocirurgia, Departamento de Neurologia; ** Setor de Endocrinologia Pediátrica, Departamento de Pediatria; *** Departamento de Radiologia. Aceite: 25-abril-1995. 
A puberdade atrasada (PA) pode ser uma condição temporária que espontaneamente se reverte ou um estado permanente de inabilidade para alcançar o desenvolvimento puberal adequado. Em meninos, PA é definida pela falta de caracteres sexuais secundários após 14 anos de idade. Pode tratar-se de uma PA constitucional (ou maturação lenta constitucional), que afeta $0,6 \%$ da população normal e que é uma condição temporária. Em contrapartida, existem os casos patológicos e permanentes de PA, que são devidos à anormalidades do eixo hipotálamo-hipófise-gônada. $\mathrm{O}$ hipogonadismo-hipogonadotrófico pode ser resultado de diferentes anomalias do sistema nervoso central (SNC). O quadro clínico depende do tempo de duração e da magnitude da deficiência hormonal, bem como da síntese e/ou liberação de outros hormônios do hipotálamo e/ou da hipófise ${ }^{18}$. A síndrome de Kallmann (SK) combina anosmia ou hiposmia (devida a aplasia ou hipoplasia dos bulbos olfatórios) com deficiência de gonadotrafinas secundária à deficiência de GnRH ${ }^{4,9}$. Há considerável heterogeneidade nesta patologia, de tal forma que podemos encontrar em uma mesma família alguns indivíduos com hipogonadismo hipogonadotrófico $(\mathrm{HH})$ e olfato normal e outros com alteração do olfato sem deficiência hormonal. A herança é autossômica recessiva ou dominante com penetrância variável e ocorre por deleção do gene localizado no Xp22.3. A incidência deste distúrbio ainda não foi estabelecida, mas em muitos serviços é citada com a principal causa de HH. Os hormônios luteinizante ( $\mathrm{LH}$ ) e folículo-estimulante (FSH) elevam-se em cerca da metade dos indivíduos com SK após estímulo agudo com $\mathrm{GnRH}^{11,13}$. O uso repetitivo por 3 ou mais dias de GnRH provoca aumento das gonadotrofinas plásmaticas em todos os pacientes com $\mathrm{SK}$, mas não em indivíduos com pan-hipopituitarismo ${ }^{16}$. O cisto aracnóide (AC) é uma coleção de fluido recoberto por membrana similar à aracnóide e representa $1 \%$ das lesões expansivas intracranianas ${ }^{15}$. A origem do AC está provavelmente relacionada à divisão ou duplicação da aracnóide ${ }^{17}$. No passado acreditava-se que ocorria uma agenesia da região fronto-temporal no período fetal ${ }^{14}$. Estudos recentes de volume cerebral em pacientes com AC demonstram não haver diminuição do volume cerebral no hemisfério com $\mathrm{AC}$, quando comparado com o hemisfério contralateral ${ }^{19}$.

Relatamos a associação de síndrome de Kallmann (SK) e cisto aracnóide em um paciente de 18 anos de idade, mostrando a investigação subsidiária, conduta e evolução do caso.

\section{RELATO DO CASO}

LF, sexo masculino, branco, 18 anos de idade, estudante da quinta série, natural e procedente de Valinhos (SP), iniciou investigação no Ambulatório de Adolescentes do Hospital das Clínicas da UNICAMP com queixa de atraso puberal e baixo crescimento após os 10-12 anos de idade (aos 10 anos, altura de $133 \mathrm{~cm}, \mathrm{z}$ score $=-0,74)$. Referia ainda, dificuldade de aprendizado e distúrbio da fala, bem como episódios esporádicos de cefaléia e vômitos. Não havia história de consanguinidade na família, nem casos semelhantes e negava queixas de hiposmia ou anosmia. Pai com estatura de $175 \mathrm{~cm}$ e mãe com $165 \mathrm{~cm}$ (estatura alvo entre 169 e $185 \mathrm{~cm}$ ). Ao exame: pesava $52 \mathrm{Kg}$ (P3-10) e media 162 cm (P3-10, com z score $=-2,17)$, aspecto discretamente eunucóide, sem ginecomastia, com pilificação pubiana discreta grau II; pênis com $5 \mathrm{~cm}$ de comprimento (GII), porém com testículos de $3-4 \mathrm{~cm}^{3} \mathrm{e}$ discreta pilificação axilar. Ao exame neurológico e neuropsicológico (bateria de testes de Luria 6 ) o paciente apresentava-se consciente, orientado, com discreta apraxia oral e manual (na

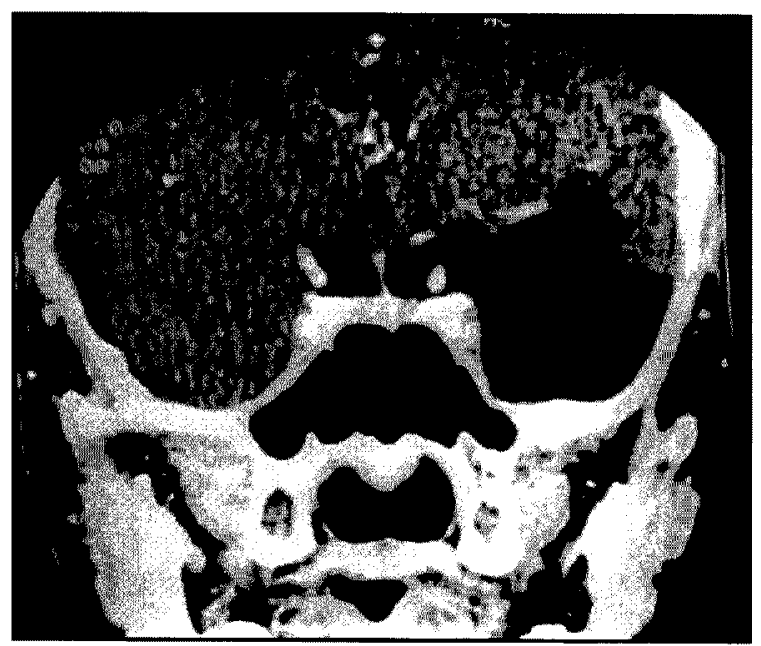

Fig 1. CT coronal pré-operatória mostrando cisto aracnóide na fossa média esquerda causando estiramento e deslocamento da haste hipofisária contralateralmente. 


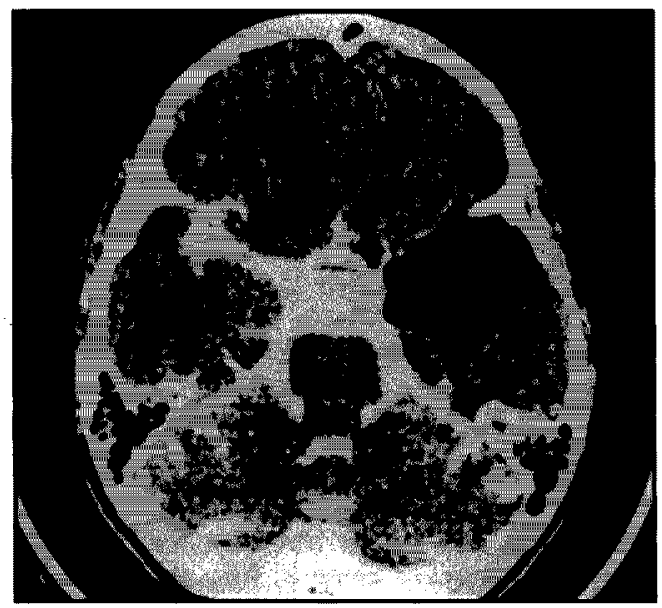

Fig 2. CT axial com metrizamida intratecal.

organização dinâmica do movimento), sem apraxia ideomotora ou construcional. Estereognosia e percepção visual-espacial normais. Fala receptiva e expressiva normais, exceto troca do " $R$ " pelo " $L$ ", como por exemplo na palavra "garfo", defeito que sempre apresentou desde criança. Leve déficit intelectual-discursivo e de aprendizado (memorização) verbal. Fundoscopia ocular normal, estrabismo convergente à direita, sem déficit motor ou sensitivo, reflexos normais.

Conclusão: Déficit de crescimento com atraso puberal provavelmente devido a patologia de SNC (regiāo hipotálamo-hipofisária)

Exames realizados - (1) $\mathrm{LH} 0,9 \mathrm{mUL} / \mathrm{mL}$ (normal, nl 1,1 a 8,2); (2) FSH 0,5 mUl/mL (normal, nl 1,43 a 11,5); (3) Prolactina 2,2 ng/mL (normal, nl 3,1 a $20,5 \mathrm{ng} / \mathrm{mL}$ ); (4) Testosterona total $<0,2$ $\mathrm{ng} / \mathrm{mL}$ (normal $\mathrm{nl}, 2,7$ a 10,7); (5) Testosterona livre $<0,6 \mathrm{ng} / \mathrm{mL}$ (normal, $\mathrm{nl}>27$ ); (6) Estradiol 5,0 pg/mL (normal $\mathrm{nl}<55$ ); (7) Idade óssea 11 anos (TW2 carpal); (8) Cariótipo = 46,XY; conclusão: hipogonadismo-hipogonadotrófico. (9) Teste GnRH agudo: LH basal 0,9 $\Delta$ 1,2; FSH basal $<0,009 \Delta$ zero. (10) Megateste (GH, TSH, cortisol e prolactina) normais. (11) Teste GnRH prolongado: $L H$ basal $<0,09 \Delta 2,7$; FSH basal 2,2 $\Delta 5,1$. (12) CT de crânio: presença de cisto aracnóide de fossa média esquerda com aparente comunicação com a cisterna supra-selar causando estiramento e discreto deslocamento da haste hipofisária para o lado contralateral (Fig 1) (13) CT (com metrizamida intratecal): presença de cisto aracnóide da fossa média esquerda não comunicante com o espaço subaracnóide ou cisterma supraselar (Fig 2).

Conclusão: Hipogonadismo-hipogonadotrófico (tipo hipotalâmico, bloqueado por problema também na hipófise) e cisto aracnóide.

Evolução terapêutica - Pelo quadro clínico-neurológico do paciente optou-se por intervenção cirúrgica, tendo sido submetido a uma derivação cisto-peritoneal com válvula de baixa pressão tipo Holter. A tomografia de controle mostrou redução do cisto com catéter dentro de sua cavidade (Fig 3). O paciente cresceu $2 \mathrm{~cm}$ em 3 meses (P10), aumentou a pilificação axilar e pubiana, sem aumento do pênis e testículos (adrenarca). Manteve LH, FSH e testosterona em níveis baixos, tendo sido introduzido terapêutica de reposição hormonal. Foi realizado teste de olfação sendo constatado anosmia bilateral. Foi submetido ainda a uma ressonância nuclear magnética que demonstrou ausência de tracto olfatório bilateralmente (Fig 4).

Conclusão: hipogonadismo-hipogonadotrófico devido à síndrome de Kallmann e cisto aracnóide.

\section{COMENTÁRIOS}

O AC é geralmente descoberto em pacientes com menos de 20 anos de idade. Em lactentes e crianças o quadro clínico predominante é a macrocrania. Já em adolescentes e

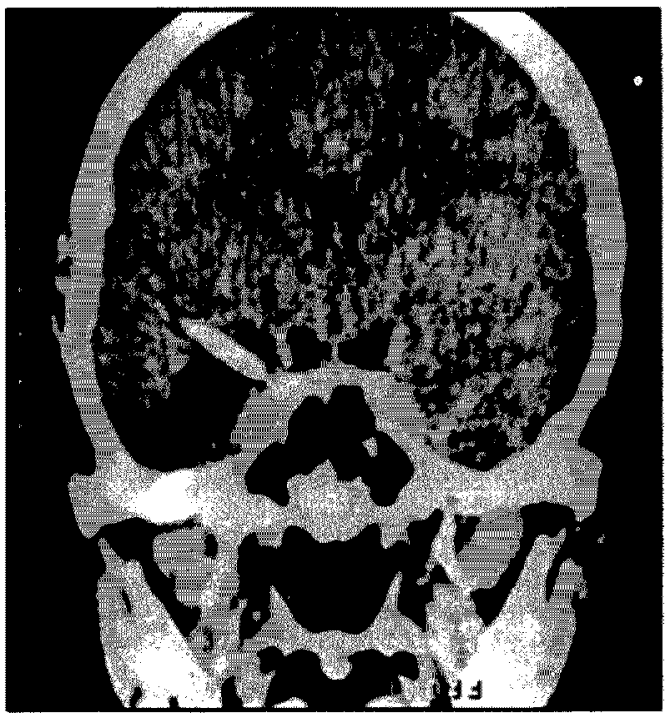
adultos, pode causar cefaléia, convulsões, Fig 3. CT coronal no pós-operatório. 


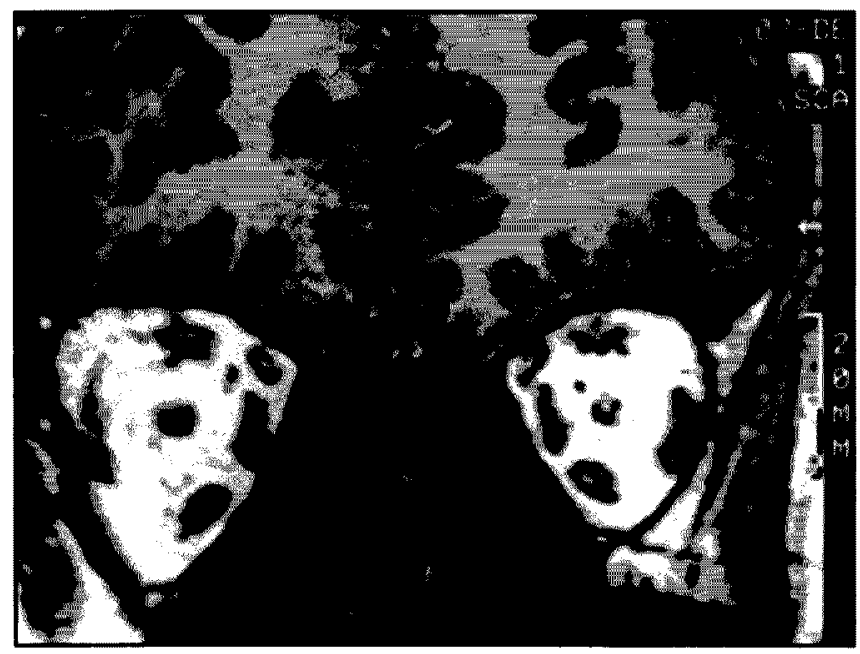

Fig 4. RNM coronal mostrando ausência de tracto olfatório bilateral (setas).

hidrocefalia, sinais e sintomas de hipertensão intracraniana, retardo mental, paralisia de nervos cranianos, sinais focais ou mesmo ser assintomático ${ }^{1,5}$.

O AC raramente está associado com distúrbio hormonal, com exceção de cistos localizados em região supra-selar quando mais frequentemente podem causar puberdade precoce, deficiência de GH, deficiência de ACTH e também a sindrome de "bobble-head doll"3,7,12. No entanto, neste caso, o cisto estava localizado na fossa média esquerda e aparentemente causava estiramento e deslocamento contralateral da haste hipofisária.

A ressonância nuclear magnética tem possibilitado o diagnóstico in vivo da SK devido à ausência ou à presença rudimentar do sulco olfatório e tracto olfatório ${ }^{\not}$.

A presença de SK associada a AC de fossa média não é descrita na literatura. Por outro lado, os resultados da avaliação hormonal inicial e com GnRH prolongado (três dias de GnRH intramuscular, $100 \mathrm{mcg} /$ dia) não é o que frequentemente se observa na SK em que poderia ser encontrada até uma resposta normal puberal de $\mathrm{LH}(\mathrm{LH}>25 \Delta \text { ou }>15)^{2,10,11,13,16}$. Na realidade, os resultados encontrados com bloqueio de LH e resposta parcial de FSH são mais sugestivos de patologia hipofisária.

A etiologia do hipogonadismo hipogonadotrófico deste caso é devida à síndrome de Kallmann. Entretanto, os resultados laboratoriais não completamente compatíveis, podem estar mascarados neste caso pela associação inusitada desta síndrome com uma patologia de fossa média como o cisto aracnóide, com repercussão compressiva na região selar e supra-selar.

\section{REFERÊNCIAS}

1. Aicardi J, Bauman F. Supratentorial extracerebral cysts in infants and children. J Neurol Neurosurg Psychiatry 1975, 38:57-68.

2. Bell J, Spitz I, Slonin A. Heterogeneity of gonadotropin response to LHRH in hypogonadotropic hypogonadism. J Clin Endocrinol Metab 1973, 36:791-794.

3. Binitie $O$, Williams B, Case CP. A suprasellar subarachnoid pouch: aetiological considerations. J Neurol Neurosurg Psyquiatry 1984, 47:1066-1074.

4. Boyar RM, Finkelstein JW, Witkin M. Studies of endocrine function in "isolated" gonadotropin deficiency. J Clin Endocrinol Metab 1973, 36:64-72.

5. Choux M, Raybaud C, Pinsard N, Hassoun J, Gambarelli D. Intracranial supratentorial cysts in children excluding tumor and parasite cysts. Child Brain 1978, 4:15-32. 
6. Christensen AL. Luria's neuropsychological investigation. Copenhagen:Munksgaard, 1979.

7. Di Rocco C. Arachnoid cysts. In Youmans JR (ed). Neurological surgery.Ed 3. Philadelphia: W. B. Saunders, 1990, p 1299-1325.

8. Klingmuiller D, Dewe W, Krahe T, Brecht G, Scheikert H. Magnetic resonance imaging of the brain in patients with anosmia and hypothalamic hypogonadism (Kallmann's syndrome). J Clin Endocrinol Metab 1987, 65:581-584.

9. Lieblich JM, Rogol AD, White BJ. Syndrome of anosmia with hypogonadotropic hypogonadism (Kallmann's syndrome): clinical and laboratory studies in 23 cases. Am J Med 1982, 73:506-519.

10. Marshall JC, Harsoulis P, Anderson DC. Isolated pituitary gonadotrophin deficiency: gonadotrophin secretion after synthetic luteinizing hormone and follicle stimulating hormone-releasing hormone. Br Med $J 1972$, 4:643-645.

11. Oettinger M, Bruneteau DW, Pasaroudakis A. FSH and LH response to LHRF in Kallmann's syndrome. Obstetr Gynecol 1976, 47: 233-236.

12. Pierre-Kahn A, Capelle L, Brauner R, Sainte-Rose C, Renier D, Rappaport R, Hirsch JF. Presentation and management of suprasellar arachnoid cysts. J Neurosurg 1990, 73:355-359.

13. Reitano JF, Caminos-Torres R, Snyder PJ. Serum LH and FSH responses to the repetitive administration of gonadotropin-releasing hormone in patients with idiopatic hypogonadotropic hypogonadism. J Clin Endocrinol Metab 1975, 41:1035-1042.

14. Robinson RG. The temporal lobe agenesia syndrome. Brain 1964, 87:87-106.

15. Robinson RG. Congenital cysts of the brain: arachnoid malformation. Progr Neurol Surg 1971, 4:133-174.

16. Snyder PJ, Rudenstein RS, Gardner DF. Repetitive infusion of gonadotropin-releasing hormone distinguishes hypothalamic from pituitary hypogonadism. J Clin Endocrinol Meiab 1979, 48:864-868.

17. Starkman SP, Brown TC, Linell EA. Cerebral arachnoid cysts. J Neuropathol Exp Neurol 1958, 17:484-500.

18. Styne DM. Puberty and its disorders in boys. Endocrinol Metab Clin North Am 1991, 20:43-70.

19. Van der Meché FGA, Braakman R. Arachnoid cysts in the middle fossa:cause and treatment symptoms. J Neurol Neurosurg Psychiatry 1983, 46:1102-1107. 